\title{
Opinión de Profesores Titulares y Catedráticos de Universidad acerca de criterios y estándares para la acreditación del profesorado universitario
}

\author{
Juan Carlos Sierra*, Gualberto Buela-Casal*, María Paz Bermúdez \\ Sánchez*, Pablo Santos-Iglesias*
}

\begin{abstract}
Resumen: Este estudio descriptivo analiza la importancia que Profesores Titulares de Universidad y Catedráticos de Universidad de España otorgan a distintos indicadores de evaluación docente, examinándose además las diferencias entre ambos cuerpos docentes. Una muestra representativa de 1.294 Profesores Titulares y Catedráticos de Universidad españoles respondieron a una encuesta enviada por correo electrónico. Los resultados señalan que los indicadores más valorados tienen que ver con la investigación y muestran escasas diferencias entre ambos cuerpos docentes, lo que supone una ventaja para la fiabilidad y objetividad del proceso de acreditación y concurso de acceso a los cuerpos de funcionarios docentes universitarios en España.
\end{abstract}

Palabras clave: acreditación docente, profesorado universitario, cuerpo docente.

\section{Opinion of Associate Professors and Full Professors regarding the criteria and standards for the accreditation of Spanish university teachers}

\begin{abstract}
This descriptive study analyses and compares the importance given by Associate Professors and Full Professors of Spanish universities to different academic evaluation indicators. A representative sample consisting of 1294 Associate Professors and Full Professors from Spain answered a survey sent to them by e-mail. The results show that the most valued indicators are related to research work and reveal little difference between the criteria of the two study groups. The latter is an advantage with respect to the reliability and objectivity of the accreditation process and selection of tenured university professors in Spain.
\end{abstract}

Keywords: accreditation of university professors, university professorate, academic body.

\footnotetext{
* Facultad de Psicología. Universidad de Granada, España. Correo-e: jcsierra@ugr.es. Recibido: 11-09-2008; 2. ${ }^{a}$ versión: 20-11-2008.
} 


\section{Introducción}

En España la evaluación de la calidad de las universidades ha cobrado especial relevancia en los últimos años debido al proceso de adaptación al Espacio Europeo de Educación Superior (EEES) (Buela-Casal, 2005b). Esta evaluación la realizan en España once agencias autonómicas y tres grandes agencias nacionales, que focalizan su actividad evaluadora en los programas, profesores, alumnos, publicaciones, organización y uso de datos (Buela-Casal, 2005a). En este contexto es fundamental la evaluación del profesorado universitario, ya que una adecuada selección de los docentes asegura un nivel alto de calidad y eficacia de la institución universitaria (De la Orden, 1990) y contribuye a alcanzar los objetivos de la universidad de forma global (Edwards, 2006). Tal y como afirma BuelaCasal (2005a), "una universidad puede tener excelentes instalaciones, los mejores medios, los mejores alumnos, pero si no tiene un cuerpo de profesores con nivel de excelencia es casi lo mismo que no tener nada" (p. 314).

Uno de los temas más controvertidos en la evaluación del profesorado universitario tiene que ver con los criterios de evaluación y el acuerdo existente sobre cuáles deben ser y su valor o importancia. Éste ha sido uno de los pilares fundamentales en las críticas y revisiones sobre el antiguo sistema de habilitación nacional y el actual sistema de acreditación del profesorado universitario en España (Buela-Casal, 2005a, 2007a, 2007b; De la Orden, 1990; Saravia, 2004). En estas revisiones se sostiene que no se puede garantizar la sistematicidad y objetividad de la evaluación y acreditación del profesorado universitario a menos que se publiquen previamente los criterios, indicadores y estándares de manera operativa.

En España se han realizado diversos intentos por clarificar los criterios de evaluación del profesorado universitario (Buela-Casal y Sierra, 2007; Chacón, Pérez-Gil, Holgado y Lara, 2001; Pulido y Pérez, 2003; Saravia, 2004). Chacón y colaboradores realizaron un proceso de validación de contenido con la ayuda de 17 jueces expertos y encontraron que los indicadores más importantes de evaluación de la calidad universitaria se agrupan en tres bloques principales: enseñanza, investigación y gestión universitaria, bloques que han sido señalados por diversos autores (De Miguel, 1998; García-Valcárcel, 2001; Pulido y Pérez, 2003) y que se observan también en el "Programa de Evaluación del Profesorado" elaborado por la Agencia Nacional de Evaluación de la Calidad y Acreditación (ANECA) (2005), en el "Sistema de Evaluación del Profesorado para su Contratación por las Universidades de Madrid" (Agencia de Calidad, Acreditación y Prospectiva de las Universidades de la Comunidad de Madrid ACAP, 2006) e incluso en el borrador del nuevo "Estatuto del Personal Docente e Investigador" publicado recientemente en España por el Ministerio de Ciencia e Innovación (2008). Otra propuesta realizada con la intención de clarificar más los criterios para la evaluación del profesorado universitario fue desarrollada por Buela-Casal y Sierra (2007). Utilizando una muestra representativa de Profesores Titulares de Universidad (PTU) y Catedráticos de Universidad (CU) de España, analizaron la importancia que 
éstos dan a distintos indicadores para la acreditación del profesorado universitario, así como el número mínimo que se debe poseer para poder ser acreditado como PTU o CU. Los resultados principales mostraron que los indicadores a los que más importancia se da son a los relacionados con la investigación, en concreto a la publicación de artículos en revistas del Journal Citation Reports (JCR). No obstante, se encontraron diferencias entre distintas áreas de conocimiento, ya que en las áreas de Artes y Humanidades y Ciencias Sociales y Jurídicas lo que más se valora son los libros publicados en editoriales de reconocido prestigio, frente a las áreas de Ciencias, Ciencias de la Salud e Ingeniería y Arquitectura, donde el indicador más valorado es la publicación de artículos en revistas del JCR. Resultados similares fueron encontrados por Saravia (2004).

Todos estos estudios son de inestimable utilidad, pues el conocimiento de los criterios e indicadores, además de su valor, va a permitir no sólo que las evaluaciones sean más objetivas y transparentes, sino que los miembros que forman parte de las comisiones de evaluación sean nombrados también gracias a un proceso más objetivo, facilitando además las posibles reclamaciones (Buela-Casal, 2005a, 2007a, 2007b; De la Orden, 1990). A esto se debe añadir que conocer cuál es la valoración que se hace de esos criterios en función del área de conocimiento permite una evaluación más justa y adecuada de los candidatos; tal como sostiene Buela-Casal (2007a) «el peso de cada criterio debe ser establecido previamente, y además su valor podría variar en función del campo de conocimiento" (pp. 6-7), lo que puede hacerse extensivo a las distintas titulaciones e incluso cuerpos docentes. Por tanto, con el objetivo de conocer las diferencias en la importancia que Profesores Titulares de Universidad y Catedráticos de Universidad dan a distintos indicadores de evaluación del profesorado universitario, se realiza el presente estudio descriptivo (Montero y León, 2007), en el que se han seguido en la medida de lo posible las normas de Ramos-Álvarez, Moreno-Fernández, Valdés-Conroy y Catena (2008).

\section{Método}

\subsection{Muestra}

Los participantes fueron seleccionados de forma aleatoria a partir del listado definitivo de sorteables para formar parte de las pruebas de habilitación nacional, correspondientes a la convocatoria de 21/09/2005 (Ministerio de Educación y Ciencia, 2005). El listado estaba compuesto por 28.770 profesores funcionarios, de los cuales se seleccionaron al azar, en cada área de conocimiento establecida por el Consejo de Coordinación Universitaria, un 20\% de los Catedráticos de Universidad y un 20\% de los Profesores Titulares de Universidad o Catedráticos de Escuela Universitaria (CEU), lo que ofreció un total de 5.754 profesores funcionarios seleccionados para el estudio. Teniendo en cuenta los participantes 
seleccionados que no respondieron la encuesta, la muestra final estuvo formada por 1.294 profesores universitarios de los cuerpos docentes de Profesores Titulares de Universidad y Catedráticos de Universidad de España con, al menos, un tramo de investigación los primeros y dos tramos los segundos. La muestra seleccionada permite trabajar con un nivel de confianza del $95 \%$ y con un error de estimación del 3\%. El 71,5\% de la muestra fueron PTU $(n=926)$ y el $28,5 \%$ CU $(n=368)$. La media de edad de los CU fue 54,15 $(D T=7,81)$, mientras que la de los PTU fue 45,61 $(D T=7,16)$. Por último, los CU tienen una media de 3,70 tramos de investigación $(D T=1,27)$ y los PTU $1,98(D T=0,95)$.

\subsection{Instrumento}

Se elaboró un cuestionario ad hoc formado por dos partes. La primera consta de varias preguntas en las que se recoge información del participante sobre su edad, sexo, universidad a la que pertenece, número de tramos de investigación que tiene acreditados, cuerpo docente al que pertenece, titulación en la que imparte la mayoría de su docencia, área y rama de conocimiento. La segunda está formada por 42 ítems que hacen referencia a distintos criterios o indicadores utilizados en el proceso de evaluación del profesorado universitario, según la Agencia de Calidad, Acreditación y Prospectiva de las Universidades de la Comunidad de Madrid ACAP (2006) y la Agencia Nacional de Evaluación de la Calidad y Acreditación ANECA (2005), a los que se responde indicando el peso o importancia de dicho indicador a la hora de evaluar a un candidato en una prueba de acreditación. Se responde a través de una escala tipo Likert que oscila entre 0 (nada importante) y 10 (muy importante). Los ítems que componen el cuestionario se corresponden con los 42 indicadores que aparecen en la Tabla I.

\subsection{Procedimiento}

Los datos se obtuvieron a través de una encuesta realizada por correo electrónico. Así, después de seleccionar al $20 \%$ de la población de PTU y CU $(n=5.754)$ se buscaron las direcciones de correo electrónico de cada uno de los participantes seleccionados. Una vez obtenidas las direcciones se envió por correo electrónico una breve justificación del estudio, la identificación de los autores y una invitación a participar en el mismo, suministrando para ello un enlace a una página web de acceso a la encuesta y un código único de acceso a la página, de manera que se garantizaba el anonimato de los participantes, la confidencialidad de las respuestas y el acceso único y privado a la encuesta de los receptores de los correos. Las respuestas se almacenaron de forma automática y ordenada en una hoja de datos Excel para su posterior análisis. 


\section{Resultados y discusión}

Se analizó en primer lugar cuáles son los indicadores más valorados en ambos cuerpos docentes. Los resultados muestran que los más importantes para los PTU son, en este orden: artículos publicados en revistas del JCR, dirección de proyectos de investigación con financiación externa, libros publicados en editoriales de reconocido prestigio, asignaturas impartidas en enseñanza reglada y dirección de tesis doctorales defendidas. Mientras que los CU, en este orden, valoran más: artículos publicados en revistas del JCR, asignaturas impartidas en enseñanza reglada, dirección de proyectos de investigación con financiación externa, libros publicados en editoriales de reconocido prestigio y dirección de tesis doctorales defendidas (véase la Tabla I). Es decir, los indicadores más valorados son los mismos en ambos cuerpos docentes, aunque no en el mismo orden.

Estos resultados replican, parcialmente, los encontrados por Buela-Casal y Sierra (2007) y por Pulido y Pérez (2003), ya que en ambos estudios revelaron que los indicadores más valorados tienen que ver con las publicaciones -en revistas del JCR en el caso del trabajo de Buela-Casal y Sierra-, proyectos de investigación y las asignaturas o clases impartidas. La diferencia fundamental estriba en que Pulido y Pérez no encontraron diferencias en función de los campos de conocimiento considerados. Resultados similares se observan cuando se analizan los indicadores más comunes en la elaboración de ranking académicos internacionales (Buela-Casal, Gutiérrez-Martínez, Bermúdez-Sánchez y Vadillo-Muñoz, 2007), pues los resultados demuestran que el indicador más frecuente y con mayor peso en los diferentes ranking analizados son los artículos en bases de datos del ISI (Institute for Scientific Information). De la misma manera, sistemas de evaluación bien establecidos reconocen la mayor importancia de las publicaciones en revistas con sistema de revisión por pares, y más concretamente en revistas del JCR (véanse los criterios de la Agencia de Calidad, Acreditación y Prospectiva de las Universidades de la Comunidad de Madrid ACAP, 2006; y de la Agencia Nacional para la evaluación de la Calidad y Acreditación ANECA, 2005). Obviamente, aunque los indicadores más relevantes estén relacionados con la investigación, esto no quiere decir que hablar de excelencia universitaria se reduzca a hablar de excelencia investigadora (Pulido, 2005). De hecho - y a la vista de los resultados- es imposible obviar por completo la labor docente, ya que un elevado porcentaje de profesores universitarios considera que las asignaturas impartidas están relacionadas con sus trabajos de investigación (Grupo Helmántica, 1999) y, además, la preparación de profesores para el EEES debe centrarse más en la docencia y no tanto en la investigación (González Soto, 2005)

En segundo lugar, se analizó si existían diferencias entre PTU y CU en la valoración que hacían de los distintos criterios. En la Tabla I se muestran también los resultados de la prueba $t$ de Student realizada sobre el peso dado a cada indicador tomando como factor el cuerpo docente. Los resultados muestran escasas diferencias entre Profesores Titulares de Universidad y Catedráticos de 


\section{TABLA 1}

Comparación del peso de cada indicador en función del cuerpo docente

\begin{tabular}{c|c|c|c|c|c}
\hline Criterios e indicadores & $\begin{array}{c}\text { Cuerpo } \\
\text { docente }\end{array}$ & $M$ & $D T$ & $t$ & $p$ \\
\hline
\end{tabular}

\section{Formación}

Formación académica

\begin{tabular}{ll|c|c|c|c|l}
\hline $\begin{array}{l}\text { 1. Segundas o más titulaciones no exigi- } \\
\text { das como requisito mínimo }\end{array}$ & $\begin{array}{c}\text { CU } \\
\text { PTU }\end{array}$ & $\begin{array}{l}2,40 \\
2,16\end{array}$ & $\begin{array}{l}2,27 \\
2,19\end{array}$ & 1,75 & 0,07 \\
\hline 2. Premios extraordinarios y menciones & $\begin{array}{c}\text { CU } \\
\text { PTU }\end{array}$ & $\begin{array}{l}3,56 \\
3,50\end{array}$ & $\begin{array}{l}2,46 \\
2,69\end{array}$ & 0,35 & 0,72 \\
\hline Formación complementaria \\
\hline $\begin{array}{l}\text { 3. Programas de especialización (MIR, } \\
\text { BIR, PIR y equivalentes; especialista y } \\
\text { master universitarios) }\end{array}$ & CU & $\begin{array}{l}3,78 \\
3,51\end{array}$ & $\begin{array}{l}2,60 \\
2,49\end{array}$ & 1,71 & 0,08 \\
\hline 4. Cursos de postgrado & CU & $\begin{array}{l}3,48 \\
3,81\end{array}$ & $\begin{array}{l}2,56 \\
2,79\end{array}$ & 1,98 & $0,04^{*}$ \\
\hline
\end{tabular}

\section{Experiencia}

Experiencia docente

Enseñanza impartida por año

\begin{tabular}{|c|c|c|c|c|c|}
\hline 5. Asignaturas en enseñanza reglada & $\begin{array}{l}\text { CU } \\
\text { PTU }\end{array}$ & $\begin{array}{l}6,99 \\
7,24\end{array}$ & $\begin{array}{l}2,56 \\
2,57\end{array}$ & 1,59 & 0,11 \\
\hline $\begin{array}{l}\text { 6. Tareas docentes universitarias (becario } \\
\text { tipo FPI, tutor UNED) }\end{array}$ & $\begin{array}{l}\text { CU } \\
\text { PTU }\end{array}$ & $\begin{array}{l}4,22 \\
4,39\end{array}$ & $\begin{array}{l}2,58 \\
2,66\end{array}$ & 1 & 0,31 \\
\hline 7. Clases en máster o cursos de postgrado & $\begin{array}{l}\text { CU } \\
\text { PTU }\end{array}$ & $\begin{array}{l}5,12 \\
5,40\end{array}$ & $\begin{array}{l}2,63 \\
2,70\end{array}$ & 1,67 & 0,09 \\
\hline 8. Cursos de formación & $\begin{array}{c}\mathrm{CU} \\
\mathrm{PTU}\end{array}$ & $\begin{array}{l}3,30 \\
3,66 \\
\end{array}$ & $\begin{array}{l}2,39 \\
2,54 \\
\end{array}$ & 2,33 & $0,02^{*}$ \\
\hline \multicolumn{6}{|l|}{ Otros méritos docentes } \\
\hline $\begin{array}{l}\text { 9. Dirección académica de trabajos (pro- } \\
\text { yectos fin de carrera, tesinas, DEA, me- } \\
\text { morias de máster) }\end{array}$ & $\begin{array}{l}\text { CU } \\
\text { PTU }\end{array}$ & $\begin{array}{l}5,52 \\
5,70\end{array}$ & $\begin{array}{l}2,71 \\
2,66\end{array}$ & 1,08 & 0,27 \\
\hline $\begin{array}{l}\text { 10. Proyectos de innovación y mejora do- } \\
\text { cente financiados }\end{array}$ & $\begin{array}{l}\text { CU } \\
\text { PTU }\end{array}$ & $\begin{array}{l}4,18 \\
4,42\end{array}$ & $\begin{array}{l}2,66 \\
2,77\end{array}$ & 1,39 & 0,16 \\
\hline $\begin{array}{l}\text { 11. Evaluaciones positivas de encuestas de } \\
\text { alumnos }\end{array}$ & $\begin{array}{l}\text { CU } \\
\text { PTU }\end{array}$ & $\begin{array}{l}4,13 \\
4,63\end{array}$ & $\begin{array}{l}3,05 \\
3,10\end{array}$ & 2,62 & $0,009^{* *}$ \\
\hline
\end{tabular}

Experiencia investigadora

Investigaciones realizadas

\begin{tabular}{l|c|c|c|c|c}
\hline 12. Dirección de proyectos de investiga- & CU & 7,74 & 2,34 & 3,53 & $0,000^{* * * *}$ \\
ción con financiación externa & PTU & 7,21 & 2,63 & 3,53 \\
\hline
\end{tabular}


TABLA I (continuación)

\begin{tabular}{|c|c|c|c|c|c|}
\hline Criterios e indicadores & $\begin{array}{l}\text { Cuerpo } \\
\text { docente }\end{array}$ & $\boldsymbol{M}$ & $D T$ & $t$ & $p$ \\
\hline $\begin{array}{l}\text { 13. Participación proyectos de investiga- } \\
\text { ción con financiación externa }\end{array}$ & $\begin{array}{l}\text { CU } \\
\text { PTU }\end{array}$ & $\begin{array}{l}6,23 \\
6,45\end{array}$ & $\begin{array}{l}2,54 \\
2,56\end{array}$ & 1,37 & 0,16 \\
\hline $\begin{array}{l}\text { 14. Dirección proyectos financiados por la } \\
\text { propia universidad }\end{array}$ & $\begin{array}{l}\text { CU } \\
\text { PTU }\end{array}$ & $\begin{array}{l}4,13 \\
4,41\end{array}$ & $\begin{array}{l}2,51 \\
2,60\end{array}$ & 1,72 & 0,08 \\
\hline $\begin{array}{l}\text { 15. Participación proyectos financiados por } \\
\text { la propia universidad }\end{array}$ & $\begin{array}{l}\text { CU } \\
\text { PTU }\end{array}$ & $\begin{array}{l}3,18 \\
3,84\end{array}$ & $\begin{array}{l}2,40 \\
2,60\end{array}$ & 4,35 & $0,000^{* * *}$ \\
\hline \multicolumn{6}{|l|}{ Actividades relacionadas con la investigación } \\
\hline $\begin{array}{l}\text { 16. Dirección de tesis doctorales defen- } \\
\text { didas }\end{array}$ & $\begin{array}{l}\text { CU } \\
\text { PTU }\end{array}$ & $\begin{array}{l}6,98 \\
6,64\end{array}$ & $\begin{array}{l}2,70 \\
2,66\end{array}$ & 1,99 & $0,04^{*}$ \\
\hline \multicolumn{6}{|l|}{ Experiencia profesional } \\
\hline $\begin{array}{l}\text { 17. Actividades en puestos de dirección o } \\
\text { gestión, puestos técnicos o profesionales }\end{array}$ & $\begin{array}{l}\text { CU } \\
\text { PTU }\end{array}$ & $\begin{array}{l}3,85 \\
3,65\end{array}$ & $\begin{array}{l}2,66 \\
2,56\end{array}$ & 1,23 & 0,21 \\
\hline $\begin{array}{l}\text { 18. Colaboraciones puntuales o informes } \\
\text { profesionales }\end{array}$ & $\begin{array}{l}\text { CU } \\
\text { PTU }\end{array}$ & $\begin{array}{l}2,97 \\
2,93\end{array}$ & $\begin{array}{l}2,29 \\
2,35\end{array}$ & 0,26 & 0,79 \\
\hline
\end{tabular}

\section{Producción académico-científica}

\section{Publicaciones}

Artículos en revistas

\begin{tabular}{|c|c|c|c|c|c|}
\hline $\begin{array}{l}\text { 19. En Journal Citation Reports (JCR) del } \\
\text { ISI }\end{array}$ & $\begin{array}{l}\text { CU } \\
\text { PTU }\end{array}$ & $\begin{array}{l}8,31 \\
8,11\end{array}$ & $\begin{array}{l}2,27 \\
2,29\end{array}$ & 1,45 & 0,14 \\
\hline 20. En listados nacionales similares al JCR & $\begin{array}{l}\text { CU } \\
\text { PTU }\end{array}$ & $\begin{array}{l}5,29 \\
5,38\end{array}$ & $\begin{array}{l}2,87 \\
2,82\end{array}$ & 0,45 & 0,64 \\
\hline 21. Otros & $\begin{array}{l}\text { CU } \\
\text { PTU }\end{array}$ & $\begin{array}{l}3,13 \\
3,32\end{array}$ & $\begin{array}{l}2,68 \\
2,68\end{array}$ & 1,12 & 0,26 \\
\hline \multicolumn{6}{|l|}{ Libros y capítulos de libro } \\
\hline $\begin{array}{l}\text { 22. Libro en editorial de reconocido pres- } \\
\text { tigio }\end{array}$ & $\begin{array}{l}\text { CU } \\
\text { PTU }\end{array}$ & $\begin{array}{l}7,38 \\
7,06 \\
\end{array}$ & $\begin{array}{l}2,52 \\
2,71\end{array}$ & 1,98 & $0,04^{*}$ \\
\hline $\begin{array}{l}\text { 23. Libro en editorial sin reconocido pres- } \\
\text { tigio }\end{array}$ & $\begin{array}{l}\mathrm{CU} \\
\mathrm{PTU}\end{array}$ & $\begin{array}{l}3,70 \\
3,92\end{array}$ & $\begin{array}{l}2,60 \\
2,55\end{array}$ & 1,39 & 0,16 \\
\hline $\begin{array}{l}\text { 24. Capítulo de libro en editorial de reco- } \\
\text { nocido prestigio }\end{array}$ & $\begin{array}{l}\mathrm{CU} \\
\mathrm{PTU}\end{array}$ & $\begin{array}{l}5,75 \\
5,68\end{array}$ & $\begin{array}{l}2,68 \\
2,70\end{array}$ & 0,37 & 0,70 \\
\hline $\begin{array}{l}\text { 25. Capítulo de libro en editorial sin reco- } \\
\text { nocido prestigio }\end{array}$ & $\begin{array}{l}\text { CU } \\
\text { PTU }\end{array}$ & $\begin{array}{l}2,67 \\
3,07\end{array}$ & $\begin{array}{l}2,40 \\
2,44\end{array}$ & 2,62 & $0,009^{* *}$ \\
\hline \multicolumn{6}{|l|}{ Contribuciones a congresos } \\
\hline 26. Congreso internacional & $\begin{array}{l}\text { CU } \\
\text { PTU }\end{array}$ & $\begin{array}{l}4,92 \\
5,44 \\
\end{array}$ & $\begin{array}{l}2,72 \\
2,64\end{array}$ & 3,12 & $0,002^{* *}$ \\
\hline 27. Congreso nacional & $\begin{array}{l}\mathrm{CU} \\
\mathrm{PTU}\end{array}$ & $\begin{array}{l}3,27 \\
3,93\end{array}$ & $\begin{array}{l}2,44 \\
2,54\end{array}$ & 4,24 & $0,000^{* * *}$ \\
\hline
\end{tabular}


TABLA I (continuación)

\begin{tabular}{|c|c|c|c|c|c|}
\hline Criterios e indicadores & $\begin{array}{l}\text { Cuerpo } \\
\text { docente }\end{array}$ & M & $D T$ & $\boldsymbol{t}$ & $p$ \\
\hline \multicolumn{6}{|l|}{ Medios específicos de producción y difusión } \\
\hline 28. Traducciones de libros & $\begin{array}{l}\text { CU } \\
\text { PTU }\end{array}$ & $\begin{array}{l}3,01 \\
2,95\end{array}$ & $\begin{array}{l}2,45 \\
2,47\end{array}$ & 0,36 & 0,71 \\
\hline 29. Exposiciones artísticas o documentales & $\begin{array}{l}\mathrm{CU} \\
\mathrm{PTU}\end{array}$ & $\begin{array}{l}2,49 \\
2,51\end{array}$ & $\begin{array}{l}2,37 \\
2,47\end{array}$ & 0,08 & 0,96 \\
\hline $\begin{array}{l}\text { 30. Producciones de radio, televisión o } \\
\text { cine }\end{array}$ & $\begin{array}{l}\text { CU } \\
\text { PTU }\end{array}$ & $\begin{array}{l}2,30 \\
2,31\end{array}$ & $\begin{array}{l}2,35 \\
2,44\end{array}$ & 0,07 & 0,94 \\
\hline \multicolumn{6}{|l|}{ Registros en la propiedad industrial e intelectual } \\
\hline $\begin{array}{l}\text { 31. Patentes o modelos de utilidad y regis- } \\
\text { tros en la propiedad intelectual }\end{array}$ & $\begin{array}{l}\mathrm{CU} \\
\mathrm{PTU}\end{array}$ & $\begin{array}{l}5,44 \\
5,31\end{array}$ & $\begin{array}{l}3,08 \\
3,11\end{array}$ & 0,69 & 0,49 \\
\hline \multicolumn{5}{|l|}{ Movilidad } & \\
\hline $\begin{array}{l}\text { 32. Estancias docentes y de investigación } \\
\text { en España }\end{array}$ & $\begin{array}{l}\mathrm{CU} \\
\mathrm{PTU}\end{array}$ & $\begin{array}{l}4,21 \\
4,47 \\
\end{array}$ & $\begin{array}{l}2,34 \\
2,48\end{array}$ & 1,69 & 0,09 \\
\hline $\begin{array}{l}\text { 33. Estancias docentes y de investigación } \\
\text { en el extranjero }\end{array}$ & $\begin{array}{l}\text { CU } \\
\text { PTU }\end{array}$ & $\begin{array}{l}6,80 \\
6,60\end{array}$ & $\begin{array}{l}2,76 \\
2,82\end{array}$ & 1,13 & 0,25 \\
\hline
\end{tabular}

\section{Gestión universitaria y otros méritos}

Gestión universitaria

Desempeño de cargos unipersonales

\begin{tabular}{|c|c|c|c|c|c|}
\hline $\begin{array}{l}\text { 34. Dirección/coordinación/secretaría de } \\
\text { acciones formativas (másters y docto- } \\
\text { rados) }\end{array}$ & $\begin{array}{l}\text { CU } \\
\text { PTU }\end{array}$ & $\begin{array}{l}3,98 \\
4,01\end{array}$ & $\begin{array}{l}2,65 \\
2,71\end{array}$ & 0,21 & 0,83 \\
\hline 35. Organización de reuniones científicas & $\begin{array}{l}\text { CU } \\
\text { PTU }\end{array}$ & $\begin{array}{l}4,29 \\
4,23\end{array}$ & $\begin{array}{l}2,66 \\
2,61\end{array}$ & 0,37 & 0,70 \\
\hline 36. Rectorado y Vicerrectorado & $\begin{array}{l}\text { CU } \\
\text { PTU }\end{array}$ & $\begin{array}{l}3,78 \\
3,68\end{array}$ & $\begin{array}{l}3,06 \\
3,08\end{array}$ & 0,53 & 0,59 \\
\hline 37. Decanato, Vicedecanato y similares & $\begin{array}{l}\text { CU } \\
\text { PTU }\end{array}$ & $\begin{array}{l}3,37 \\
3,38\end{array}$ & $\begin{array}{l}2,77 \\
2,85\end{array}$ & 0,02 & 0,97 \\
\hline $\begin{array}{l}\text { 38. Dirección de Departamento, Secretario } \\
\text { y similares }\end{array}$ & $\begin{array}{l}\text { CU } \\
\text { PTU }\end{array}$ & $\begin{array}{l}3,33 \\
3,22\end{array}$ & $\begin{array}{l}2,72 \\
2,76\end{array}$ & 0,68 & 0,49 \\
\hline \multicolumn{6}{|l|}{ Otros méritos } \\
\hline $\begin{array}{l}\text { 39. Miembro de consejo editorial en revis- } \\
\text { ta ISI }\end{array}$ & $\begin{array}{l}\text { CU } \\
\text { PTU }\end{array}$ & $\begin{array}{l}5,26 \\
4,77\end{array}$ & $\begin{array}{l}2,93 \\
2,90\end{array}$ & 2,69 & $0,007^{* * *}$ \\
\hline $\begin{array}{l}\text { 40. Miembro de consejo editorial en revis- } \\
\text { ta no ISI }\end{array}$ & $\begin{array}{l}\text { CU } \\
\text { PTU }\end{array}$ & $\begin{array}{l}2,90 \\
2,89\end{array}$ & $\begin{array}{l}2,36 \\
2,29\end{array}$ & 0,06 & 0,94 \\
\hline
\end{tabular}


TABLA I (continuación)

\begin{tabular}{|c|c|c|c|c|c|}
\hline Criterios e indicadores & $\begin{array}{l}\text { Cuerpo } \\
\text { docente }\end{array}$ & $\boldsymbol{M}$ & $D T$ & $t$ & $p$ \\
\hline 41. Director de revista en ISI & $\begin{array}{l}\mathrm{CU} \\
\mathrm{PTU}\end{array}$ & $\begin{array}{l}6,40 \\
5,80\end{array}$ & $\begin{array}{l}3,13 \\
3,23\end{array}$ & 3 & $0,003^{* *}$ \\
\hline 42. Director de revista en no ISI & $\begin{array}{l}\mathrm{CU} \\
\mathrm{PTU}\end{array}$ & $\begin{array}{l}3,63 \\
3,60\end{array}$ & $\begin{array}{l}2,57 \\
2,65\end{array}$ & 0,19 & 0,84 \\
\hline
\end{tabular}

${ }^{*} p<0,05 ;{ }^{* *} p<0,01 ;{ }^{* * *} p<0,001$.

Nota: MIR (Médico Interno Residente); BIR (Biólogo/Bioquímico Interno Residente); PIR (Psicólogo Interno Residente); FPI (Formación de Personal Investigador); UNED (Universidad Nacional de Educación a Distancia); DEA (Diploma de Estudios Avanzados).

Universidad; sólo en 12 de los 42 indicadores, lo que supone un desacuerdo en el 28,57\% de ellos. Las diferencias muestran que los PTU dan más importancia a los cursos de postgrado, cursos de formación, evaluaciones positivas en encuestas de alumnos, participación en proyectos de investigación financiados por la propia universidad, capítulos de libros publicados en editoriales de reconocido prestigio y contribuciones en congresos internacionales y nacionales. Por su parte, los CU dan más importancia a la dirección de proyectos de investigación con financiación externa, dirección de tesis doctorales leídas, publicación de libros en editoriales de reconocido prestigio, ser miembro del consejo editorial de una revista del $J C R$ y ser director de una revista del JCR.

Este análisis de las diferencias entre PTU y CU muestra un acuerdo en el $71,43 \%$ de los indicadores, que implica una ventaja para la formación de las comisiones de acreditación, su funcionamiento y los resultados de las mismas. Es decir, una comisión formada por PTU y CU realizará una evaluación más justa y coherente si los miembros que la componen están de acuerdo en el valor de los criterios e indicadores, resolviendo una de las principales críticas al actual sistema de acreditación en España (Buela-Casal, 2007b; De la Orden, 1990). En los casos en los que existen diferencias entre ambos cuerpos docentes, los PTU valoran más los cursos de postgrado, cursos de formación, evaluaciones positivas en encuestas de alumnos, la participación en proyectos de investigación financiados por la universidad, los capítulos de libro publicados en editoriales sin reconocido prestigio y las contribuciones a congresos nacionales y a congresos internacionales. Por su parte, los CU dan más importancia a la dirección de proyectos de investigación con financiación externa, dirección de tesis doctorales defendidas, libros publicados en editoriales de reconocido prestigio, ser miembro del comité editorial de una revista del JCR y ser director de una revista del JCR. Estas diferencias señalan que los CU conceden más importancia a aquellos indicadores que son más difíciles de alcanzar y que están asociados, generalmente, a una trayectoria académica más avanzada. 


\section{Conclusiones}

Los resultados del presente estudio ponen de manifiesto algunos aspectos importantes que pueden ayudar a mejorar y hacer más objetivo el sistema de acreditación a cuerpos de funcionarios docentes universitarios. Primero, la importancia de clarificar los criterios de evaluación, que supone una ventaja doble, ya que permite conocer los indicadores por los que se va a evaluar a un candidato y permite orientar la carrera académica hacia aquello que se evalúa (BuelaCasal, 2005a; Pulido, 2005). Esto, que se ha tratado en el presente trabajo y en otros anteriores (Buela-Casal y Sierra, 2007; Pulido y Pérez, 2003; Saravia, 2004; Sierra, Buela-Casal, Bermúdez y Santos-Iglesias, en prensa) en relación al profesorado universitario, también se hace extensivo a otros aspectos de la evaluación de la calidad académica como los criterios e indicadores para la obtención de la Mención de Calidad en Programas de Doctorado (Buela-Casal y Castro, 2008; Novo y Arce, 2007) o los criterios para la evaluación de la calidad universitaria (Pagani et al., 2006). Segundo, conocer el valor que tienen los distintos indicadores contribuye a tener un sistema de evaluación más objetivo y transparente (Buela-Casal, 2007b; De la Orden, 1990). En este sentido, una de las propuestas más interesantes y útiles ha sido la desarrollada por Buela-Casal y Sierra (2007), que supone una aportación difícil de cuestionar ya que recoge la opinión de la comunidad científica universitaria en España, en la que, además, se analiza el peso de cada indicador diferenciando entre campos de conocimiento. Por último, el hecho de diferenciar entre PTU y CU tiene la ventaja de recoger la opinión de dos sectores distintos y poder contemplar las diferencias entre ambos cuerpos en función de quién sea el candidato en la evaluación.

A pesar de las ventajas que suponen estudios como el presente, todavía faltan muchos intentos y reflexiones para hacer que la evaluación de la calidad universitaria sea cada vez más objetiva y esté mejor definida, centrándose para ello en los múltiples factores que la componen: profesorado, programas de estudios, alumnos, instituciones y organización, entre otros.

\section{Agradecimientos}

Estudio financiado por la Dirección General de Universidades (Referencia: EA2006-0017).

\section{Referencias}

Agencia de Calidad, Acreditación y Prospectiva de las Universidades de la Comunidad de Madrid ACAP (2006): Sistema de evaluación del profesorado para su contratación por las Universidades de Madrid. Madrid: ACAP. 
Agencia Nacional de Evaluación de la Calidad y Acreditación ANECA (2005): Programa de evaluación de Profesorado: principios y orientaciones para la aplicación de los criterios de evaluación. Recuperado el 13 de noviembre de 2006, de http://www. aneca.es/modal eval/pep nuevo orientaciones.html

Buela-Casal, G. (2005a): El Sistema de Habilitación Nacional: criterios y proceso de evaluación. Análisis y Modificación de Conducta, 31, 313-346.

Buela-Casal, G. (2005b): La evaluación de la calidad en el proceso de convergencia europea. Revista Mexicana de Psicología, 22, 306-314.

Buela-Casal, G. (2007a): Consideraciones metodológicas sobre el procedimiento de acreditación y del concurso de acceso a cuerpos de funcionarios docentes universitarios. Revista Electrónica de Metodología Aplicada, 12, 1-14.

Buela-Casal, G. (2007b): Reflexiones sobre el sistema de acreditación del profesorado funcionario de Universidad en España. Psicothema, 19, 473-482.

Buela-Casal, G., y Castro, A. (2008): Criterios y estándares para la obtención de la Mención de Calidad en Programas de Doctorado: evolución a través de las convocatorias. International Journal of Psychology and Psychological Therapy, 8, 127-136.

Buela-Casal, G.; Gutiérrez-Martínez, O.; Bermúdez-Sánchez, M. P., y Vadillo-Muñoz, O. (2007): Comparative study of international academic rankings of universities. Scientometrics, 71, 349-365.

Buela-Casal, G., y Sierra, J. C. (2007): Criterios, indicadores y estándares para la acreditación de profesores titulares y catedráticos de Universidad. Psicothema, 19, 537-551.

Chacón, S.; Pérez-Gil, J. A.; Holgado, F. P., y Lara, A. (2001): Evaluación de la calidad universitaria: validez de contenido. Psicothema, 13, 294-301.

De la Orden, A. (1990): Evaluación, selección y promoción del profesorado universitario. Revista Complutense de Educación, 1, 11-29.

De Miguel, F. M. (1998): La evaluación del profesorado universitario: criterios y propuestas para mejorar la función docente. Revista de Educación, 315, 67-83.

Edwards, K. (2006): New approaches to staff recruitment in Higher Education. Higher Education in Europe, 31, 289-293.

García-Valcárcel, A. (2001): La función docente del profesor universitario, su formación y desarrollo profesional. En A. García-Valcárcel (Coord.), Didáctica universitaria (pp. 1-27). Madrid: La Muralla.

González Soto, A. P. (2005): Posibilidades de formación en el Espacio Europeo de Educación Superior. En J. Cabero (Ed.), Formación del profesorado universitario para la incorporación del aprendizaje en red en el EEES. Sevilla: Universidad de Sevilla.

Grupo Helmántica (1999): Características profesionales de los docentes universitarios de Castilla y León. Salamanca: Junta de Castilla y León.

Ministerio de Ciencia e Innovación (2008): Estatuto del Personal Docente e Investigador. Recuperado el 11 de noviembre de 2008, de http://web.micinn.es/01_Portada/01Ministerio/031Prensa/00@Prensa/101108a.pdf.

Ministerio de Educación y Ciencia (2005): Habilitación para el acceso a los cuerpos funcionarios docentes universitarios. Resolución lista definitiva. Recuperado 13 de junio de 2006, de http://www.mec.es/educa/ccuniv.

Montero, I. y León, O. G. (2007): A guide for naming research studies in Psychology. International Journal of Clinical and Health Psychology, 7, 847-862. 
Novo, M. y Arce, R. (2007): Sesgos en la evaluación de la Mención de Calidad en programas de doctorado. En M. P. Bermúdez \& A. Castro (eds.), Evaluación de la calidad delaEducación SuperiorydelaInvestigación (IVForo). Recuperado 7 de febrerode 2008, de http://feugr.ugr.es/pags/cursos/IVForo/LibroResumenesIVForo.pdf

Pagani, R.; Vadillo, O.; Buela-Casal, G.; Sierra, J. C.; Bermúdez, M. P.; Gutiérrez-Martínez, O., et al. (2006): Estudio internacional sobre criterios e indicadores de calidad de las universidades. Madrid: ACAP.

Pulido, A. (2005): Indicadores de calidad en la evaluación del profesorado universitario. Estudios de Economía Aplicada, 23, 667-684.

Pulido, A., y Pérez, J. (2003): Propuesta metodológica para la evaluación de la calidad docente e investigadora: planteamiento y experimentación. Madrid: Instituto L. R. Klein-Centro Stone.

Ramos-Álvarez, M. M.; Moreno-Fernández, M. M.; Valdés-Conroy, B., y Catena, A. (2008): Criteria of the peer review process for publication of experimental and quasi-experimental research in Psychology: A guide for creating research papers. International Journal of Clinical and Health Psychology, 8, 751-764.

Saravia, M. A. (2004): Evaluación del profesorado universitario. Un enfoque desde la competencia profesional. Tesis doctoral no publicada, Universidad de Barcelona.

Sierra, J. C.; Buela-Casal, G.; Bermúdez, M. P., y Santos-Iglesias, P. (2009): Diferencias por sexo en los criterios y estándares e productividad científica y docente en profesores funcionarios en España. Psicothema, 21, 124-132. 